\title{
Jean-Michel Salanskis: la literatura desde las matemáticas"
}

\author{
Jean-Michel Salanskis: Literature from \\ Mathematics
}

M. ${ }^{a}$ Paz CePedello

Departamento de Ciencias del lenguaje

Facultad de Filosofía y Letras

Universidad de Córdoba

Plaza del Cardenal Salazar, s/n. Córdoba, 14071

fezcemom@uco.es

Orcid ID 0000-0002-0480-4394
RECIBIDO: 19 DE SEPTIEMBRE DE 2017 ACEPTADO: 21 DE DICIEMBRE DE 2017
Resumen: El objetivo de este trabajo es analizar la reflexión de Jean-Michel Salanskis sobre las relaciones posibles entre la literatura y las matemáticas. Para ello, en primer lugar, estableceremos en qué consisten las operaciones de axiomatización y formalización, procedentes del campo de las matemáticas, para, en un segundo momento, valorar el interés que puede tener su aplicación en la consideración y comprensión del hecho literario. La propuesta de Salanskis no deja de tener una dimensión comparatista, muy fructífera en el último medio siglo, que pone en relación, en este caso, los métodos de análisis e interpretación de dos disciplinas muy alejadas entre sí, para mostrar, si es posible, que no se encuentran tan distantes.

Palabras clave: Salanskis. Axiomatización. Formalización. Matemáticas. Literatura.

\begin{abstract}
The aim of this study is to analyse JeanMichel Salanskis' critical reflection on the possible relationships between literature and mathematics. On the one hand, we will establish what the axiomatization and formalization operations consist of, originating from the field of mathematics. On the other hand, we are going to consider the interest that the application of these operations might have in the consideration and understanding of the literary fact. Salanskis's proposal has a fruitful comparative dimension, especially in the last half century, which, in this case, relates the methods of analysis and interpretation of two disciplines very different from each other in order to show, if possible, that they are not so distant.
\end{abstract}

Keywords: Salanskis. Axiomatization. Formalization. Mathematics. Literature.

\footnotetext{
* Este trabajo se ha realizado en el marco del proyecto de investigación "Actualidad de la hermenéutica" (FFI2013-41662-P), financiado por el Ministerio de Economía y Competitividad para el periodo 2014-2018.
} 


\section{INTRODUCCIÓN: SOBRE JEAN-MiCHEL SALANSKIS}

L

a interesante propuesta de la que nos vamos a ocupar en este trabajo se la debemos a quien es, en la actualidad, profesor de Filosofía de la Ciencia, Lógica y Epistemología en la Universidad Paris X-Nanterre, pero cuyas primeras investigaciones se desarrollan en el campo de las matemáticas puras, donde trabaja en la Teoría de los grupos finitos con Claude Chevalley. J. M. Salanskis comienza su vinculación con la filosofía de la mano de Lyotard por esos mismos años, finales de los 70 y principios de los 80 , lo que le lleva a ingresar en el CNRS como filósofo y desarrollar una intensa y extensa reflexión centrada en la Hermenéutica. Algunos títulos nos dan cuenta de esta fructífera dedicación. Baste recordar L'Herméneutique formelle de 1991 (y reeditada más recientemente, en 2013), Heidegger (1997), Husserl (1998) y, muy especialmente, Sens et philosophie du sens (2001) y Territoires du sens (2007). Salanskis parte de la constatación de que la filosofía contemporánea se ha interesado por el sentido más que nunca, especialmente después de lo que se dio en llamar "el giro lingüístico", ${ }^{1}$ para poner de manifiesto la necesidad de desarrollar lo que él denomina "filosofía del sentido": asumiendo que la filosofía no se encuentra directa y puramente al servicio exclusivo de la verdad, como la ciencia, y que tampoco ha de estar sometida a la búsqueda del Ser, como toda su tradición invita a pensar, el francés apuesta por una filosofía que parta de la búsqueda del sentido del sentido, una comprensión del sentido de inspiración levinasiana que privilegia la llamada y no la flecha intencional. ${ }^{2}$

1. Este giro, de gran acogida en los inicios del siglo pasado, hunde sus raíces en el empirismo de Locke y de Hume. Sus principales representantes afirman que la verdad es una cuestión de palabras, no de las ideas (ver Soler Peña 9).

2. Junto a estos planteamientos que vertebran, sobre todo, la obra de 2001, Territoires $d u$ sens, a partir de lo que entonces denominó programa "etho-analitico", propone algunas concreciones. Separa, así, tres regiones del sentido, a saber: la política, el amor y el sujeto, para las que emplea una terminología propia, esto es, tres "ethos". Se trata de estratos de comportamiento colectivo que se estudian como formas capaces de empujar cierto sentido director. Cada vez que una "palabra de idealidad" nos llama a la invención y producción de un mundo compartido que le corresponde en tanto que desempeña el papel de "solicitante", nuestros comportamientos son testigos de una comprensión compartida de aquello que es requisito para que el sentido dirigido por ese solicitante no caiga en el olvido. La "semilla" del ethos en causa es el conjunto de cláusulas que mantienen y relanzan el sentido de cada región. Los capítulos de Territoires du sens pretenden describir las tres regiones mencionadas. Dichos capítulos explicitan aquello a lo que nos comprometemos al pretender iniciar una relación amorosa (cap. 2), al exhibir un determinado posicionamiento político (cap. 1) o al esforzarnos en inscribirnos como sujeto en el tiempo y con respecto a los otros (cap. 3). Estos conceptos van a resultar de extraordinario interés, sobre todo, en las escasas pero sagaces reflexiones que Salanskis lleva a cabo sobre el funcionamiento de la ficción de lo que nos ocuparemos en un trabajo específico al respecto. 
Sin embargo, su formación inicial en el campo de las matemáticas puras no solo nunca fue abandonada, sino que, muy al contrario, ha sido eficazmente aprovechada en su reflexión filosófica: recordemos que formuló una descripción heideggeriana del pensamiento matemático y una concepción husserliana de los fundamentos de las matemáticas, a lo que habría que sumar la aparición en 2008 de la conocida y aplaudida Philosophie des mathématiques. Esta dimensión de carácter interdisciplinar de su obra y de su pensamiento está, además, más que justificada si atendemos al tiempo que Salanskis permanece integrado en el equipo de Jean Petitot, ${ }^{3}$ en la unidad dirigida por Marc Barbut. $^{4}$

Este recorrido somero que acabamos de hacer por la trayectoria de Jean Michel Salanskis nos parecía necesario antes de adentrarnos en el objetivo de este trabajo, que es, como reza su título, la literatura vista desde las matemáticas. En este sentido, vale recordar que el filósofo ya había explorado territorios que, de alguna manera, colindaban con los temas vertebrales de su reflexión: la tradición judía como elemento fundamental, en el siglo XXI, para el pensamiento a partir del cruce entre ciencia y filosofía; la pérdida de sentido de la izquierda europea; los usos contemporáneos de la fenomenología o los modelos cognitivos como esquemas a partir de los cuales pueda explicarse la tarea hermenéutica. Pero el hecho literario, como tal y aisladamente, en contadas ocasiones había sido objeto específico de su tarea filosófica. ${ }^{5}$ Es en el año 2013 cuando aparece, en la revista Les Temps modernes, un artículo titulado "La littérature entre axiomatique et formalisme". En él, Salanskis parte de la confusión frecuente en la idea de "forma-

3. Jean Petitot, como muestra Carlos Francisco Soler Peña, en su trabajo de tesis La objetividad matemática según fean Petitot, ha intentado integrar, en su doble condición de filósofo y matemático, dos tradiciones filosóficas: la fenomenología husserliana y la tradición lógica que debe ser reinterpretada (10).

4. Marc Barbut marcó profundamente, en los años 60, el campo de las matemáticas aplicadas a las ciencias humanas y sociales. Entre sus numerosos méritos está la fundación de la revista Matemáticas y ciencias humanas, cuyos números, a lo largo de más de cincuenta años, resultaron un excelente escaparate del proceso de aparición y consolidación de este campo disciplinar anclado en la renovación conjunta de las ciencias sociales y las matemáticas "modernas" y asociado a la ola estructuralista iniciada por el encuentro entre Levi-Strauss y André Weil en 1959. Este campo, pues, debe mucho a Marc Barbut, que fue un apasionado promotor y una figura clave al tiempo que advertía de los posibles excesos y malentendidos, como puso de manifiesto en su conocido artículo "Sur le sens du mot structure en mathématiques" (1966).

5. A modo de ejemplo se puede citar, entre estas incursiones en el terreno específicamente literario, la publicación en el año 2007 del artículo "Fiction des mondes" en la revista Alliage, en un número dedicado al funcionamiento de la ciencia ficción. 
lismo" de dos operaciones bien diferentes: la operación de axiomatización y la operación de formalización. A partir de esta confusión, el francés desarrolla una interesante reflexión sobre las relaciones entre la literatura y las matemáticas.

Para avanzar sobre su propuesta procederemos, siguiendo el itinerario que el filósofo diseña, del siguiente modo: en primer lugar intentaremos definir en qué consisten esas operaciones mencionadas de axiomatización y formalización procedentes del campo de las matemáticas para, en un segundo momento, reflexionar sobre el interés que puede tener su aplicación en la consideración y comprensión del hecho literario. La propuesta de Salanskis no deja de tener una dimensión comparatista, muy fructífera en el último medio siglo, que pone en relación, en este caso, los métodos de análisis e interpretación de dos disciplinas muy alejadas entre sí, para mostrar, si es posible, que no se encuentran tan distantes. ${ }^{6}$

\section{EL ESTRUCTURALISMO MATEMÁTICO: ENTRE LA AXIOMATIZACIÓN Y LA FORMALIZACIÓN}

El filósofo que nos ocupa parte de una doble acepción del concepto de axioma y, consecuentemente, del proceso de axiomatización: una procedente del campo de la geometría y otra, más jugosa para Salanskis según veremos, del álgebra. ${ }^{7}$ Los fundamentos de la geometría de David Hilbert (1899), como reconstrucción de una disciplina que se había mantenido prácticamente inalterada desde su nacimiento de la mano de Euclides, ${ }^{8}$ es el punto de partida para explicar la primera acepción de axiomatización en la medida en que el mate-

6. Al respecto, es preciso recoger aquí, por su valiosa aportación en lo que a las relaciones entre las matemáticas y otras disciplinas -especialmente teoría literaria- se refiere, los trabajos de Robert Thomas (2000 y 2002).

7. Es preciso llamar la atención sobre la diferente naturaleza de estos dos campos matemáticos en el sentido en que ya Gauss lo formulara de modo explícito en la primera mitad del siglo XIX: dentro del vasto campo matemático y en virtud de su origen epistemológico, es preciso distinguir aquellas disciplinas que tienen una dimensión exclusivamente mental (llamadas matemáticas puras, entre las que se encuentran la aritmética, el álgebra, etc.) de aquellas que tienen su origen, al menos en parte, en la experiencia (matemáticas mixtas, entre las que están la geometría o la mecánica). Gauss escribe a Besell el 9 de abril de 1830: "Debemos humildemente admitir que, mientras el número es solo un producto de nuestro pensamiento, el espacio tiene además una realidad fuera de nuestro pensamiento, a la cual no podemos prescribirle a priori sus leyes" (ver Giovannini 125).

8. Recordemos que hasta el siglo XIX la obra de Euclides, Elementos, se seguía utilizando como libro de texto fundamental en los estudios de geometría. 
mático alemán llevó a cabo en la obra citada, en palabras de Eduardo N. Giovannini (122):

una presentación axiomática de la geometría euclídea [que] trajo aparejada una nueva manera de entender la naturaleza de las teorías geométricas y matemáticas en general, que logró capturar magistralmente el creciente impulso hacia la abstracción y la sistematización que venía dominando la matemática desde la segunda mitad del siglo XIX. ${ }^{9}$

Se trataba, en definitiva, de situar esta rama de las matemáticas en un sistema deductivo propio a partir de una lista de axiomas, que ya no son verdades evidentes sino intuiciones poderosas, sobre los elementos "punto", "recta" y "plano", entre otros, cuyos significados ya no se discuten porque pueden ser sustituidos, sino que lo que interesa son sus relaciones definidas. Estamos ante lo que se conoce como "axiomatización fundacional".

Pero a Salanskis le resulta más útil, porque además lo considera más corriente dentro del campo de las matemáticas, otro uso de la expresión "axiomatizar". Para explicar esta segunda acepción recurre al trabajo llevado a cabo por Kolmogórov en relación con la teoría de la probabilidad a partir de la teoría de conjuntos -recordemos que ahora nos encontramos en el campo del álgebra y no de la geometría-. El matemático soviético propone un sistema de axiomas a partir de una serie de "eventos" que generalizaba el marco clásico de la probabilidad, el cual obedece a la regla de cálculo de casos favorables sobre casos posibles. Como el filósofo francés especifica, ya se hacía cálculo de probabilidades desde Pascal, pero estas aproximaciones no permitían deducir todos los teoremas y resultados importantes que hoy forman parte de la teoría de la probabilidad. A partir de los trabajos de Kolmogórov se define el contexto de base necesario para el despliegue matemático probabilista como "espacios de sucesos" o "espacios de probabilidades". Como consecuencia de lo dicho los "contextos de base" con los que Pascal trabajaba son reidentificados como casos particulares.

A partir de este ejemplo, se puede afirmar que el proceso de axiomatización consistiría en designar y definir nuevos "eventos" por la exigencia de un cierto número de propiedades, es decir, en introducir unas categorías de even-

9. Este "creciente impulso" no impide, en ningún caso, el reconocimiento de que la génesis de la historia de la abstracción y la formalización se encuentra en la modernidad filosófica en su vertiente racionalista, como muestran los trabajos de S. Krämer (1988 y 1991). 
tos. Este proceso lleva aparejado, necesariamente, un nuevo lenguaje, lo que Salanskis llama "subidioma del lenguaje matemático", empleado para las nuevas categorías y eventos y que va a suponer el planteamiento de nuevos problemas. Estos "actos de axiomatización", tal y como se ha definido en su segunda acepción, tienen el valor de "reinstitución heurística" de un campo del trabajo matemático: ${ }^{10}$ por un lado, se reconocen "eventos" -antes denominados elementos- ya conocidos como casos de nuevas categorías y, por otro, se reformulan unas propiedades ya establecidas con un nuevo lenguaje.

En realidad, si nos detenemos en estas dos acepciones y reflexionamos sobre ellas desde una perspectiva ajena a los campos de las matemáticas utilizados para definirlas, caeremos en la cuenta de que ambas maneras de caracterizar el "proceso de axiomatización" no se encuentran demasiado alejadas en la medida en que las dos, la primera de manera implícita y la segunda de manera explícita, requieren el uso de un lenguaje específico. Y es, precisamente, este hecho el que puede explicar la confusión, de la que Salanskis nos advierte al principio de su ensayo, entre la operación de axiomatizar y la de formalizar.

Para explicar el proceso de formalización, Salanskis, después de definirlo como "introducir algo en el marco de un lenguaje formal" $(2013,180)$, recuerda la tarea llevada a cabo por Frege al respecto. ${ }^{11}$ La publicación en el año 1879 de Conceptografía o Escritura de conceptos marcó el inicio de una nueva era dentro de las matemáticas: a partir de la idea de que era posible aplicar las leyes de la lógica a la aritmética, el alemán introduce en esta una nueva sintaxis que permitió formalizar una enorme cantidad de nuevos argumentos. Además, distinguió la dimensión formal de las leyes de la lógica de su contenido semántico. Como consecuencia de esta ingente labor, y a pesar de sufrir la casi total indiferencia de sus contemporáneos, en la primera mitad del siglo XX se

10. Vale recordar que Kolmogórov desarrolló un sistema axiomático que supone el pilar básico de la teoría de las probabilidades a partir de la teoría de conjuntos.

11. Salanskis no se remonta, quizá por considerarlo obvio, a las incalculables y pioneras aportaciones de Descartes o Leibniz en este campo. El primero, como se sabe, criticó duramente la lógica tradicional y los silogismos. Frente a ellos, propone una nueva concepción del saber que se alcanzaría mediante el empleo de un método que genere verdades fundadas como las que ofrecen las matemáticas. Se propone, entonces, expandir el modelo matemático a todas las ciencias para transformarlas y dotarlas de certidumbre. Leibniz, por su parte, persigue la formalización del saber, esto es, dotar a la filosofía de un instrumento que le permita alcanzar el mismo rigor científico que las matemáticas. Para ello crea una lógica simbólica de carácter calculístico, en analogía con los procedimientos matemáticos, que evite el empleo del lenguaje ordinario y la ambigüedad de sus términos. 
llevó a cabo la formalización de las matemáticas en su totalidad, la teoría formal de conjuntos de Zermelo/Fraenkel/Skolem. ${ }^{12}$

La formalización, entendida como proceso, resulta ser, según Salanskis, el establecimiento de un lenguaje no natural, donde se fijan unos símbolos y las posibles relaciones entre ellos, que se denominarán "fórmulas". Se establece, pues, no solo un nuevo lenguaje, sino una nueva textualidad en la medida en que, si los símbolos y sus potenciales uniones dan lugar a fórmulas, la técnica de derivación de estas fórmulas a través de la expresión precisa generará "teoremas":

Avant la formalisation, l'activité démonstrative, en mathématique, est supposée avoir cours au sein du langage naturel, du langage partagé. Après la formalisation, il apparaît que, pour que quelque chose puisse être considéré comme une preuve correcte, il faut que cela ait été couché dans un langage formel et que cela obéisse aux règles de la dérivation dans un tel langage. (Salanskis 2013, 181)

Salanskis se cuida muy mucho de emplear el término axioma en su explicación del proceso de formalización cuando, en cualquier estudio que se consulte al respecto, por supuesto de carácter especializado, se menciona explícitamente el uso de axiomas en el trabajo llevado a cabo por Frege y sus sucesores. Es obvio que al filósofo francés le interesa mantener estos dos conceptos bien separados aunque él mismo reconocerá más adelante la interdependencia entre las dos operaciones descritas, que encarnan lo que podría llamarse el estructuralismo matemático, al afirmar:

Axiomatisation et formalisation, cela dit, se croisent et se superposent à un endroit absolument fondamental, celui de la réinstitution de la mathématique comme mathématique formelle ensembliste: l'instauration de la théorie ZFC, évoquée plus haut, comme référence juridique; une ins-

12. La teoría de conjuntos es una rama de la matemática cuyo propósito es estudiar unas entidades llamadas conjuntos, aunque, además, esta teoría es reconocida como los fundamentos mismos de las matemáticas. La teoría de conjuntos fue desarrollada por el alemán Georg Cantor a finales del XIX. El problema con el que se enfrentaba el matemático era la necesidad de un lenguaje específico que posibilitara la expresión de su teoría de manera más precisa. Frege llevó a cabo el primer intento, al aplicar las leyes de la lógica para fundamentar adecuadamente la teoría de conjuntos ( $\mathrm{y}$, por ende, todas las matemáticas). La paradoja descubierta por B. Russell que parecía desbaratar el sistema de Frege fue eliminada por Zermelo y, poco después, por Skolem y Fraenkel, al reducir la teoría de conjuntos a un sistema axiomático más restringido que el utilizado por Frege. 
tauration qui est à la fois axiomatisation fondationnelle et formalisation. (Salanskis 2013, 182)

A partir de la instauración de la matemática formal, Salanskis percibe esta disciplina ocupada en describir un misterioso "universo de conjuntos", más allá de la lista de axiomas de Zermelo/Fraenkel/Skolem, donde es posible encontrar y situar todos los objetos de los que hablan las matemáticas. Considerado de manera aislada, el proceso de formalización implicaría que las matemáticas no serían más que un juego "formal" de pruebas que no hay necesidad de vincular con la realidad o, dicho de otra manera, de considerarlas "aplicadas". Pero la dimensión axiomática que hemos visto incluida en la operación de formalización permite conservar nuestra noción intuitiva de conjunto y esto conduce a entender la formulación de la teoría formal de ZFC como "introducción ficcional" de un mundo. A partir de aquí se preguntará Salanskis, tras mencionar algunos conceptos tradicionalmente vinculados al ámbito de la literatura, por la posibilidad de aplicar estas operaciones en la consideración de esta práctica artística.

\section{¿ES POSIBLE LA AXIOMATIZACIÓN EN LITERATURA?}

De las dos operaciones que acabamos de explicar, sucintamente, es la primera, la axiomatización, la que el filósofo considera más directamente vinculada con el "ejercicio literario". En concreto, piensa en la axiomatización en su segunda acepción, en la medida en que, al igual que la teoría de Zermelo/Fraen$\mathrm{kel} / \mathrm{Skolem}$, la literatura proyecta un universo ficcional. Es preciso, pues, detenerse en esta sugerente propuesta.

En primer lugar, se parte de la necesaria diferenciación entre la ficción axiomática y la ficción literaria para después ponerlas en relación. Así Salanskis escribe:

Ce qu'on appelle axiomatisation des espaces topologiques, des variétés différentielles ou des espaces probabilisés, introduit, via la définition interne à la théorie des ensembles d'une catégorie, une population arbitraire d'objets ou de configurations, dont la mathématique va ensuite explorer la diversité et les comportements: il n'est pas excessif ici de parler d'un monde des espaces topologiques ou des variétés différentielles. $(2013,184)$

A partir de esta afirmación, que en buena medida es hipótesis, la pregunta a la que el filósofo intentará responder es si en el ejercicio literario se da una ope- 
ración análoga a esta "axiomatización de mundos" (matemáticos), es decir, si la configuración de la ficción literaria responde a un proceso de axiomatización que abre un universo en virtud de unas características presentes en el texto. Sabemos, y él mismo lo adelanta, que ya respondió afirmativamente a esta cuestión valiéndose para ello de un ejemplo fetiche en tanto resultaba, a sus ojos, de lo más ilustrativo: Los cantos de Maldoror. ${ }^{13} \mathrm{El}$ texto de Lautrémont catapultaba a los lectores a un universo ficticio, configurado por una serie de leyes, donde tenían cabida unos elementos literarios (personajes, lugares, etc.) que se relacionan como contrarios. El conocido pasaje del canto I: "Hice un pacto con la prostitución para sembrar el desorden en las familias" (76), protagonizado por el gusano brillante y la prostituta, además del inquietante narrador, ofrece un mundo extraño que parece fundamentarse en las funciones y relaciones que se dibujan entre los personajes citados, es decir, una "axiomatización" en palabras de Salanskis. ${ }^{14}$

Pero, ¿realmente está el hecho literario "axiomatizado" en el mismo sentido en que esta operación, la de axiomatización, se entiende en matemáticas? La respuesta del filósofo será sí y no. Vayamos primero al no. En matemáticas se empieza por establecer unas reglas para el uso de ciertos conceptos (transformados en axiomas o contextualizados en la teoría de conjuntos y después sometidos a axiomas, estas son las dos acepciones de axiomatización) para, a continuación, proceder a derivar teoremas o a extraer definiciones de carácter general. Esta operación, tal cual ha sido sintetizada, no se da ni en el

13. Escrita entre 1868 y 1869 por Isidore Ducasse, más conocido por su seudónimo de Conde de Lautrémont, está considerada no solo un texto renovador de la poesía francesa del XIX, sino un libro precursor del surrealismo. Inspirado, explícitamente, por el Manfred de Byron, el Konrad de Mickiewicz y el Fausto de Goethe, Lautrémont compone una obra que, en palabras de Pérez Boitel, "deslumbra por su fuerza cognoscitiva entre el bien y el mal, en la que se debate y reside la actitud de un personaje como Maldoror, ermitaño, en ocasiones; guerrero contra las tinieblas, en otra; o buscador de tierras lejanas, pero héroe pagano, enaltecido e incomprendido". (5)

14. No podemos negar, como el mismo Salanskis reconoce, que el texto de Lautréamont, por sus particularidades estéticas, resulta especialmente útil al filósofo para la demostración de la hipótesis que persigue: "J'utilisais cet exemple, à vrai dire, comme outil pédagogique pour faire aimer les mathématiques et leurs démarches axiomatiques. Je disais à des élèves de terminale déconcertés par la définition de la notion de base de filtre, que je venais de leur introduire, qu'ils devaient prendre leur mal en patience, attendre que je leur raconte quelques histoires faisant intervenir des bases de filtre et qu'ils trouveraient l'émotion au bout" (Salanskis 2013, 185). Su generalización a otros textos construidos desde planteamientos estilísticos diferentes no es objeto de reflexión profunda en este trabajo si bien se contempla, muy pertinentemente, su aplicación a las narraciones encuadrables dentro del subgénero de la ciencia ficción, la novela histórica, la novela sentimental, entre otras, donde “j'ai le sentiment qu'il peut être largement généralisé" (Salanskis, 2013, 186). 
poema de Lautrémont ni en el hecho literario en general. El acceso a "otro mundo" que permiten los Cantos de Maldoror, por continuar con el ejemplo, no resulta de un proceso similar al descrito en matemáticas en la medida en que esa proyección no es consecuencia directa de la fundamentación de este mundo en una serie de funciones previamente establecidas. Así, Salanskis reconocerá: "la littérature procède généralement de la sorte: elle nous plonge dans l'univers fictif en le prenant d'emblée comme le réel. Il n'y a donc pas à proprement parler d'axiomatique, au sens précis de la préface axiomatique" (2013, 186).

Sin embargo, y he aquí donde responde afirmativamente, sí detecta un aspecto en común en lo que al proceso de axiomatización se refiere entre la matemática y la literatura y es el rasgo que ambas comparten de "proveedora de mundos". La manera en que Salanskis concibe el funcionamiento de la literatura y, por ende, de la ficción es de clara raíz fenomenológica, lo que es consecuente con la trayectoria de su pensamiento. Para el filósofo, la gran proeza del escritor es la de configurar un universo que ha de "convertirse en verdadero" en la mente del receptor. Para alcanzar este efecto los autores proceden de dos maneras: primero proponen un mundo, descriptiva y narrativamente, que está en su imaginación, al que los lectores acceden "sin problemas" desde la primera línea (a esto lo llama proceso) y, en segundo lugar, limitan este universo evocado con las convenciones literarias que la tradición ha fijado para cada subgénero (a esto lo llama ángulo). Es, precisamente, este "ángulo" lo que relaciona la literatura con la axiomatización en la medida en que en ella, también, está fijada de manera convencional una serie de características previas a todos los desarrollos de la escritura. Los axiomas de "género", de este modo, no solo estarían presentes en estos universos propuestos de manera más o menos implícita, sino que, además, filtran, a priori, los elementos de los que es posible, es decir, coherente, hablar en ese mundo ficticio. En este sentido, los textos literarios que pertenecen a la llamada ciencia ficción, en tanto subgénero narrativo, resultan especialmente valiosos para demostrar las afirmaciones recién expuestas:

En principe, un ouvrage de science-fiction nous place dans un monde déterminé par la donnée d'un contenu de la science future [...]. On déroule donc un monde de science-fiction en appliquant à la donnée scientifique particulière évoquée dans l'ouvre les lois de déduction de monde que l'on pense connaître: plus ou moins comme on développe les mondes topologiques et leur étude en appliquant les lois de la théorie des ensem- 
bles aux définitions de base des espaces topologiques et des applications continues. (Salanskis 2013, 187-88)

$\mathrm{Y}$ es, precisamente, en este momento de la reflexión salanskiana sobre la plausibilidad de aplicar conceptos matemáticos al análisis del hecho literario cuando el camino recorrido que nos llevaba de una disciplina a otra cambia de sentido, poniendo de manifiesto que es posible explicar el funcionamiento de las matemáticas desde la literatura o, más concretamente, desde la ficción. La narrativa no está sometida, como sabemos, a un compromiso de veracidad sino de verosimilitud, ${ }^{15}$ es decir, su objetivo es configurar un mundo imaginario con unas características (Salanskis dirá sometido a una serie de leyes), que facilitan la asunción por parte del lector de ese universo como creíble, esto es, estéticamente convincente. Sin embargo, con unas determinadas marcas formales (llámense leyes más o menos restrictivas, llámense funciones) no es suficiente. De la mano de la fenomenología hemos sabido que, para que "el efecto real" (Barthes) sea posible, es necesario que el lector "viva en primera persona" los hechos narrados. En síntesis, es preciso el proceso y el ángulo, según la terminología empleada por el filósofo. Las matemáticas, a partir de la teoría de conjuntos, pueden concebirse de la misma manera:

L'univers des ensembles, le corrélat magique de l'axiomatique ZFC, se vit comme un réel, comme un réel superlatif et luxuriant, dont le mathématicien se conçoit comme l'explorateur passionné. [...] Mon point est simplement que ma théorie générale de la satisfaction de l'exigence fictionnelle en littérature s'applique aussi à la littérature mathématique, un cas étonnant de la puissance fictionnelle humaine. (Salanskis 2013, 189)

No queremos dejar de insistir en ese curioso movimiento de las matemáticas a la literatura y viceversa que ha tenido lugar en la reflexión de Salanskis a partir del concepto de axiomatización. En ambos casos, entiéndase bien, se propone un universo ficticio como si fuera real, y así ha de ser asumido por el receptor que, en el campo de las matemáticas o en el de la ficción literaria, acepta la existencia de unas "realidades" que no puede ver ni tocar, pero que tienen un correlato real.

15. Compartimos con Pozuelo Yvancos la idea de que la categoría de verosímil es la que mejor explica la ficcionalidad literaria porque no está referida a un principio de estilización concreto, léase realismo, sino que "es por el contrario compartida por muchas formas de literatura incluyendo la más fantástica y anti-realista” (18). 


\section{LITERATURA Y FORMALIZACIÓN: LA LUCHA CON EL LENGUAJE}

Recordemos que Salanskis había definido la formalización como "le faire entrer dans le cadre d'un langage formel" $(2013,180)$ y que esta operación, aplicada a las matemáticas por Frege y sus sucesores implicaba la fijación de unos símbolos y sus potenciales relaciones entre ellos, las fórmulas, que, expresadas según técnicas precisas de derivación, daba lugar no ya a un nuevo lenguaje sino a una nueva textualidad. Parece evidente, como el mismo filósofo admite, que la tradición literaria está "marcada" por esta operación pues

chez les Grecs et les Latins, est établi le principe d'une contrainte formelle portant sur certaines unités linguistiques appelées à entrer dans des touts poétiques, les vers: ceux-ci sont définis, en substance, par une norme rythmique portant sur les longues et les brèves, et une norme de longueur. $(2013,190)$

Iguales principios formalizantes es posible encontrar en otros géneros literarios como el teatro o la novela. Sin embargo, aplicada así la formalización, parecería, en principio, que esta consistiría en un conjunto de "reglas" que se mantienen ajenas a la dimensión semántica del texto literario y estuvieran referidas a la fonética o la sintaxis. No es la operación de formalización, así entendida, la que interesa a Salanskis, sino que su reflexión sobre la aplicación de este concepto matemático al hecho literario tiene un objetivo más ambicioso y es "de savoir si, pour la littérature, le formel peut avoir un statut allant audelà de l'ornement, s'il peut être le partenaire intime de la performance de l'art littéraire" (2013, 191). El filósofo francés está pensando concretamente en las propuestas artísticas contemporáneas ${ }^{16}$ concebidas como un combate, con sus propias condiciones materiales y estructurales, siguiendo la concepción del arte, especialmente a partir de las vanguardias, preconizado por Lyotard en La Condition postmoderne (1979) y desarrollada más específicamente en su conocido trabajo "Le sublime et l'avant-garde" (1988). La pregunta que se formula Salanskis en esta línea es cómo identificar y analizar ese combate del que habla Lyotard en los textos literarios, en la medida en que estos habrán de

16. Esta idea está inspirada por J. F. Lyotard y su concepción del arte contemporáneo cuando señala que lo posmoderno es aquello que alega lo impresentable en lo moderno y en la representación misma; aquello que se niega a la consolación de las formas bellas y aboga por presentaciones nuevas no para gozar de estas, sino para hacer sentir lo que es impresentable. Lo sublime, que es lo impresentable, se caracteriza por ser en sí un contenido ausente que escapa a la claridad conceptual de la razón (ver Lyotard 1984 y 1988; Espinosa Ramírez). 
emprender una batalla con las condiciones materiales y estructurales de la expresión lingüística.

La respuesta se abre en dos direcciones: por un lado, la que presentan aquellos textos que trabajan sobre el significante para reivindicar su forma antes de que la convención lingüística lo uniera indisociablemente a un significado, que se ha impuesto sobre el significante, y, por otra parte, la de aquellas prácticas literarias que suponen un uso transgresor de la lengua. Los ejemplos para ilustrar una y otra dirección van desde las creaciones de Michel Butor o el mismo Lautrémont para la primera, a las audacias de Artaud o Joyce para la segunda. Y es que Salanskis nos recuerda, para que no olvidemos el marco teórico desde el que está llevando a cabo su reflexión, que:

Pour Lyotard, pour le dernier Lyotard tout particulièrement -mais cette tendance fut sienne depuis le début-, c'est dans ces combats que réside l'essence de l'art en général et donc de la littérature en particulier. Il lui paraît aller de soi que la tâche de la "vraie" littérature est d'enrôler les mots vers l'au-delà du système de la langue. $(2013,191)$

Pero no perdamos de vista que estas consideraciones están formuladas para intentar dilucidar la idoneidad de hablar de "formalización", aquella operación importada del campo de las matemáticas, en literatura. A Salanskis le resulta útil y, aún más diríamos, necesario el empleo de la operación que nos ocupa ahora para la consideración de la actividad literaria entendida esta como combate con el lenguaje. Así, se concibe al escritor como transgresor de una o varias "reglas" morfológicas de la lengua en la que escribe para poner de manifiesto el carácter convencional y arbitrario de la misma. Pero estas transgresiones, a fuerza de repetirse, adquieren un carácter, digamos, institucional y, por tanto, formalizado. Para ejemplificar esta idea Salanskis recurre a la obra de Tardieu y sus juegos con el lenguaje.

En realidad se podrían utilizar cientos de ejemplos similares donde los poetas intentan subvertir la relación convencional entre el significante y el significado, bien para llamar la atención sobre la forma, bien para proponer una nueva vinculación que, a la postre, lo que persigue es redescribir el mundo con un "nuevo lenguaje". Dicho así es inevitable acordarse del viejo concepto de "desautomatización" propuesto por V. Shklovski en 1917, aquel que, sintetizando enormemente, concebía el mensaje poético compuesto por una serie de artificios puestos, artísticamente, al servicio de romper nuestra recepción "automatizada" de los mensajes verbales cotidianos. Pero, junto a esto, tam- 
bién nos vemos avocados a recordar la advertencia que ya Mukařovský hacía en 1936 sobre la "estandarización" de los resultados estéticos que generan dichos artificios, cuyo valor puede quedar "caduco" con el paso del tiempo y el cambio de los contextos culturales. No olvidemos que tanto Tinianov como Mukařovský se esforzaron por que el concepto de desautomatización no fuera un principio estático ni cuantitativo. No es la suma de artificios lo que otorga la poeticidad, sino la función de los mismos. Y esta función no podría medirse únicamente frente a la convención del lenguaje cotidiano, habría de establecerse referida a las propias convenciones normativas de la tradición literaria y de las series extraliterarias. La desautomatización no era un principio absoluto, sino relativo a la función que cada elemento literario ocupa en el conjunto de normas que se actualizan, normas que, además, van variando y modificándose hasta constituir un sistema dinámico de convenciones.

Parece obvio, y así cierra Salanskis este epígrafe de su trabajo, que el concepto de formalización aplicado a la literatura resulta extraordinariamente útil incluso en aquellos textos que se proponen como "interruptores" de la obra, utilizando la terminología Lyotardiana, y del sistema de significados en el que se inscribe para sustituirlo por la pura representatividad desprovista del contenido semántico convencional ya que, finalmente, es posible extraer de estos textos una nueva serie de convenciones.

\section{CONCLUSIÓN}

Es preciso cerrar este trabajo declarando abiertamente que la reflexión salanskiana que hemos recorrido no persigue más objetivo que abundar en la siempre inquietante y jugosa relación entre la literatura y la vida:

Je reste accroché au mystère que j'ai évoqué tout à l'heure: le fait que la littérature "devrait" restituer la plausibilité du monde, qu'elle devrait nous introduire à un fictionnel qui ne diffère du réel que par l'étiquette ontologico-modale de la fiction; à un fictionnel aussi vivant que le réel ou aussi réel que la vie. Et non pas à une réalité en carton, trahissant par sa pâleur, sa pauvreté ou sa raideur qu'elle n'est pas le réel. Défi qui sonne comme impossible, pour qui respecte ce qu'il y a d'inimitable et d'inépuisable dans le réel en tant que tel. (Salanskis 2013, 194)

La comparación con el mundo matemático se le antoja al filósofo francés de extraordinaria utilidad en la medida en que le incita a reflexionar sobre si, en 
su funcionamiento, tras las operaciones de axiomatización y formalización a las que se somete a los distintos campos de la disciplina en el siglo XX, esta puede dar cuenta de la realidad. La respuesta es rotundamente positiva y la analogía con el hecho literario confirma la sospecha inicial: los mundos matemáticos, como los literarios, pueden desafiarnos, de igual manera que ocurre con el real. El desarrollo de la teoría de conjuntos llevaba aparejada la utilización, como dijimos al principio, de un lenguaje propio desprovisto de la "coloración" de la lengua natural, pero, al mismo tiempo, este hecho multiplicaba las posibilidades de expresión al infinito actual. La analogía con una concepción fenomenológica del funcionamiento de la literatura parece más que evidente en la medida en que el texto también resulta una estructura esquemática que, precisamente por este carácter, multiplica las posibilidades de interpretación y adquiere densidad en la tarea hermenéutica.

¿Se encuentra la literatura axiomatizada y formalizada en el mismo sentido en que lo están las matemáticas? Probablemente sí, pero la literatura, para Salanskis, o, al menos, las más valiosas manifestaciones literarias, solo pueden entenderse en permanente intento de huida de esas operaciones.

\section{OBRAS CITADAS}

Barbut, Marc. "Sur Le Sens du mot structure en mathématiques". Les Temps modernes 246 (1966): 791-814.

Barthes, Roland. "L'Effet de réel". Communications 11 (1968): 84-89.

Espinosa Ramírez, Luis Orlando. "La modernidad, lo sublime y un ojo cortado: nota a propósito de la estética de Jean-François Lyotard". Poliantea 6.11 (2010): 11-21.

Frege, Gottlob. Conceptografía: los fundamentos de la aritmética: otros estudios filosóficos. México: UnAM, 1972.

Giovannini, Eduardo N. "Geometría, formalismo e intuición: David Hilbert y el método axiomático formal (1891-1905)". Revista de Filosofía 39.2 (2014): 121-46.

Hilbert, David. Los fundamentos de la geometría. 1899. Madrid: CSIC, 1991.

Krämer, Sybille. Symbolische Maschinen: Die Idee der Formalisierung in geschichtlichem Abriß. Darmstadt: Wissenschaftliche Buchgesellschaft, 1988.

Krämer, Sybille. Berechenbare Vernunft: Kalkül und Rationalismus im 17. Jabrbundert. Berlin/New York: De Gruyter, 1991.

Lautrémont, Conde de. Obras completas: Los cantos de Maldoror. Poesías. Cartas. Trad. Aldo Pellegrini. Buenos Aires/Barcelona: Argonauta, 1978. 
Lyotard, Jean François. La condición posmoderna: informe sobre el saber. 1979. Trad. Mariano Antolín Rato. Madrid: Cátedra, 1984.

Lyotard, Jean François. "Le Sublime et l'avant-garde”. L'Inhumain. Paris: Galilée, 1988.

Mukařovský, Jan. "La denominación poética y la función estética del lenguaje”. 1936. Signo, función y valor: estética y semiótica del arte de Fan Mukařouský. Eds. y Trads. Jarmila Jandová y Emil Volek. Santafé de Bogotá: Plaza \& Janés, 2000. 96-104.

Pérez Boitel, Luis Manuel. "Cantos demoníacos de Maldoror o el hedónico reino de Lautrémont”. Los cantos de Maldoror. Ed. Lariza Fuentes López. Santa Clara de Cuba: Ediciones Sed de Belleza, 2006. 5-11.

Pozuelo Yvancos, José María. Poética de la ficción. Madrid: Síntesis, 1993.

Salanskis, Jean-Michel. L'Herméneutique formelle. Paris: Editions du CNRS, 1991. Salanskis, Jean-Michel. Heidegger. Paris: Les Belles Lettres, 1997.

Salanskis, Jean-Michel. Husserl. Paris: Les Belles Lettres, 1998.

Salanskis, Jean-Michel. Sens et philosophie du sens. Paris: Desclée de Brouwer, 2001.

Salanskis, Jean-Michel. Territoires du sens. Paris: Librairie Philosophique J. Vrin, 2007.

Salanskis, Jean-Michel. "Fiction des mondes". Alliage 60 (2007): 168-83.

Salanskis, Jean-Michel. Philosophie des mathématiques. Paris: Librairie Philosophique J. Vrin, 2008.

Salanskis, Jean-Michel. "La Littérature entre axiomatique et formalisme". Les Temps modernes 676 (2013): 178-97.

Shklovski, Víktor. "El arte como artificio". 1917. Teoría de la literatura. Ed. Tzvetan Todorov. Madrid: Siglo XXI, 1970. 55-70.

Soler Peña, Carlos Francisco. "La objetividad matemática según Jean Petitot". Tesis de la Universidad Nacional de Colombia. 2014. 18 de septiembre de 2017. <http://bdigital.unal.edu.co/40016/1/04438323.2014.pdf>.

Thomas, Robert. "Mathematics and Fiction I: Identification". Logique \& Analyse 171-172 (2000): 301-40.

Thomas, Robert. "Mathematics and Fiction II: Analogy". Logique \& Analyse 177-178 (2002): 185-228. 